\title{
Erratum to: Turbulent Transport of Momentum and Scalars Above an Urban Canopy
}

\author{
Linlin Wang • Dan Li · Zhiqiu Gao · Ting Sun • \\ Xiaofeng Guo · Elie Bou-Zeid
}

Published online: 31 January 2014

C Springer Science+Business Media Dordrecht 2014

\section{Erratum to: Boundary-Layer Meteorol DOI 10.1007/s10546-013-9877-z}

The original publication of the article includes some errors in Reference and citations which are corrected as given below:

1. The first paragraph of Section 1: Katul and Cl 1997; Katul et al. 1997 should be Katul et al. $1997 \mathrm{a}, \mathrm{b}$

2. The fourth paragraph of Section 1: two occurrences of Li et al. 2012 should be Li et al. $2012 \mathrm{a}$

3. The first paragraph of Section 2.2: Katul and Cl 1997; Katul et al. 1997 should be Katul et al.1997a, b

4. Above Eq.5: Katul et al. 1997; 1997 should be Katul et al. 1997a, b

5. Below Eq. 7: Katul and Cl 1997; Katul et al. 1997, 2006 should be Katul et al. 1997a, b, 2006

6. Above Eq.8: Katul and Cl 1997; Katul et al. 1997 should be Katul et al. 1997a, b

7. Above Eq: 10: Katul and Cl 1997 should be Katul et al. 1997a

8. Below Eq: 11: Katul et al. 1997; 1997 should be Katul et al. 1997a, b. twice in the same paragraph

The online version of the original article can be found under doi:10.1007/s10546-013-9877-z.

L. Wang $\cdot$ Z. Gao $\cdot$ X. Guo

State Key Laboratory of Atmospheric Boundary Layer Physics and Atmospheric Chemistry,

Institute of Atmospheric Physics, Chinese Academy of Sciences, Beijing, China

D. Li $(\varangle) \cdot$ E. Bou-Zeid

Department of Civil and Environmental Engineering, Princeton University,

Princeton, NJ 08540, USA

e-mail: danl.princeton.2009@gmail.com

T. Sun

State Key Laboratory of Hydroscience and Engineering, Department of Hydraulic Engineering, Tsinghua University, Beijing, China 
9. Below Fig. 4: Katul and Cl 1997; Katul et al. 1997 should be Katul et al. 1997a, b

10. The paragraph below Fig. 5: Katul et al. 1997 should be Katul et al. 1997b

11. The fifth and sixth paragraphs of Section 3.3: Li et al. 2012 should be Li et al. 2012a

12. The sixth paragraph of Section 3.3: Li et al. 2012 should be Li et al. 2012b

13. The correct legend for Fig. 8 is given below:

Transport efficiencies of momentum and scalars under stable (left panels) and unstable (right panel) conditions at $47 \mathrm{~m}$ (bottom panel), $140 \mathrm{~m}$ (middle panel) and $280 \mathrm{~m}$ (top panel). The vertical error bars denote the standard deviation within each bin. The solid lines are fitted functions from Bruin et al. (1993); the dashed lines are fitted functions from Quan and $\mathrm{Hu}(2009)$ and the dotted lines are fitted functions from Wood et al. (2010). The black dots denote $u$; the red diamonds denote $T$; the blue squares denote $q$ and the green dots denote $\mathrm{CO}_{2}$.

\section{Reference}

Katul G, Hsieh Cl, Kuhn G, Ellsworth D, Nie DL (1997) Turbulent eddy motion at the forest-atmosphere interface. J Geophys Res Atmos 102(D12):13409-13421 